tion, have given me special assistance in rarious ways. I am also indebted to Drs. A. E. Olpp and Natthew Steel and Messrs. Herzfeld and Bisch for cooperation and to Drs. Foster, Mosenthal and Rosenbloom for assistance. Professor Gies has given me all the facilities of his laboratory for conducting this research, as well as valuable criticism and suggestions.

Nors-In addition to the authoritics previously cited, the followin authors have published facts of interest on the subject

Astolfoni : Arch. internat. de pharm, et de therap., 1905, xiv, 59

von Fujitani: Arch. internat, de pharm. et de therap., 1905, xiv, 21.

Viley, H. W. : Bull. 84, U. S. Dept. Agric., Parts 1 to 5.

Broadway and One Hundred and Sixty-eighth Street.

\section{THE SURGICAL ELEVATION OF THE STOM- ACH IN GASTROPTOSIS BY SUTURE OF THE GASTROHEPATIC OMENTUM}

\section{A FURTHER REPORT OF RESULTS}

HENRY D. BEYEA, M.D.

Associate Surgeon, Gynecean Hospital; Associate in Gynecology, University of Pennsylvania; Assistant Gynecologist, University Hospital PHILADELPIIIA

The operation of elevation of the stomach to normal position, through either direct suture of the stomach or suture of its ligaments, is a comparatively new one in surgery.

The first description of an operation devised and practiced for this purpose was published by Duret ${ }^{1}$ of Lille, France, in 1896. The patient was greatly emaciated and in a condition described as cachectic. The operation was suggested and carried out only as a last resort, it was thought, as the only possible means of saving the patient's life. The description of this surgical procedure attracted no attention, and, although two years afterward, the operation having been performed in March, 1894, the patient had gained 25 pounds in weight and was greatly improved in health, there is no further report of a wider experience by this writer.

In the succeeding fifteen years, up to the present time, as far as can be learned from the literature, the operation has been performed by but few surgeons-Davis, Coffey, Martin, Mayo, Noble, Hodge, Clark, LaPlace and myself in this country, Rövsing in Copenhagen, Hartmann in Paris, Bier and Gelpke in Germany, and Eve in London. With the exception of Rövsing, Bier, Gelpke and myself, the experience of each surgeon has been limited to one, two or three cases. The total number of operations performed and described I have estimated to be not more than 130 , and, of these, $\% 5$ were performed by Rövsing and 26 by myself.

The operation, designated as gastropexy, which was carried out by Duret, consisted in suturing the stomach at the lesser curvature to the parietal peritoneum just below the xiphoid cartilage. The stomach was thus firmly fixed to the anterior ablominal wall.

Davis ${ }^{2}$ in 1897 sutured the gastrohepatic omentum near its attachment to the stomach to the parietal peritonemm of the abdominal wall.

Röving ${ }^{4}$ in 1899 elevated the stomach by passing three rows of interrupted sutures from right to left through the serosa and muscularis of the anterior wall of the stomach and all the structures of the abdominal

1. Invet: Rev. de chir., 1896, p. 430

2. Jaris: West. Med. Rev., October. 1897, p. 201.

4. Rövsing: A reh. f. klin. Chir., 1899, lx, 812. wall. The first row was placed just below the line of the lesser curvature of the stomach, the second row 2 $\mathrm{cm}$. below this one, and the third row $3 \mathrm{~cm}$. above the greater currature. The gastric peritoneal surface between sutures and the parietal peritoneum were scarified. When the sutures were secured a broad surface ( 6 cm.) of the anterior wall of the stomach was permanently fixed to the anterior abdominal wall near the xiphoid cartilage. Rövsing ${ }^{\overline{5}}$ recently (1906) reports a total of 75 patients operated on by this method. He has modified the operation to the extent that the three rows of sutures are placed closer together, so that the lower one-third of the stomach is left free. In 13 the operation was combined with nephropexy, and in 22 hepatopexy was also performed. He states that without exception these patients have gained good health.

Hartmann ${ }^{6}$ described a case in 1899 in which, because of dilatation and vertical dislocation of the stomach, he plicated the pyloric portion of the stomach and then fixed this end of the organ to the parietal peritoneum of the diaphragm beneath the left ribs with the plication sutures.

Coffey $^{7}$ (1902) operated in 2 cases of gastroptosis in which he elevated the stomach by suturing the great omentum at a point one inch below its attachment to the transverse colon to the anterior abdominal wall about one inch below the umbilicus.

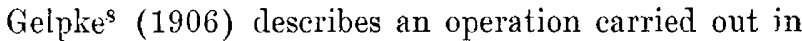
7 cases, in 5 of these combined with pyloroplasty, in which the median portion of the anterior wall of the stomach was sutured to the costal margin.

Eve $^{9}$ of London (190\%) elevated the stomach by attaching the lesser curvature to the surface of the liver at the attachment of the gastrohepatic omentum, combined with the suture of the great omentum to the anterior abdominal wall

During the years 1897 and 1898 the late Dr. William Pepper and Dr. Alfred Stengel had under their care at the University Hospital a patient suffering with the severe symptoms of gastroptosis, in which every medical and mechanical treatment, including a prolonged restcure, had been faithfully applied without benefit. At this time, March, 1898, Dr. Pepper suggested to me that some surgical operation might be undertaken to restore the stomach to normal position in this patient. I was not aware that any surgery (that of Duret and Davis) had been applied in this condition. After a careful investigation of the anatomic relations and ligamentary supports of the stomach on the cadaver, and considering the importance of the preservation of the physiologic mobility of a hollow viscus, particularly the stomach, I devised and practiced for the first time in April, 1898, the following operation:

The patient was placed on the Bolt operating-table in a position opposite to that usually employed for operations through the lower portion of the abdomen. The object of this position was to permit the elevation of the thorax and upper ablomen, which would allow the gravitation and easy displacement of the intestines and stomach out of the field of operation and give the greatest operative space, in order, in other words, to gain the every arlvantage of the Trendelenburg position. An incision about three inches in length was made through the linea alba, midway between the xiphoid cartilage and umbilicus. This incision exposed the lesser curvature and cardiac end of the stomach, the gastrohepatic ligament or

5. Rörsing: Samml, klin. Vortr. Leipsic, 1906, New Series, xv, No. 431 .

6. Hartmann. Bull et mém. Soc. de Chir. Paris, April, 1890

7. Coffey : I'hiladelphia Med. Jonl'. Oct. 11, 1902

8. (Gelpke: Arch. f. klin. Chir., Berlin, 1906, lxxx 1021

9 . Eve: Brit Med Jour. London, 1906 , i, 784-786. 
omentum, gastrophrenic ligament and the right lobe of the liver. The table was then elevated to the Trendelenburg position and the stomach displaced further downward and out of the wound by means of gauze sponges. This act caused the gastrohepatic omentum to be slightly stretched and separated from the underlying structures, which permitted an accurate determination of the length of the omentum and very much facilitated the operative manipulations. The gastrohepatic ligament was found to be composed of the two layers of delicate peritoneum, increasing in thickness and strength toward the right or pyloric end of the stomach, and above at the attachment of the liver. The gastrophrenic ligament appeared as a few thin connective tissue bands supporting the cardiac end of the stomach. Retractors were introduced and the liver held aside by placing a flat gauze sponge beneath a retractor. Three rows of interrupted silk sutures were then introduced so as to plicate and thus shorten the gastrohepatic omentum in the following manner: The first row, beginning in the gastrophrenic ligament and extending across the gastrohepatic ligament to a point almost opposite the pylorus or hepaticoduodenal ligament, was introduced so as to form a plication in the center of these ligaments, and included from above downward or vertically about $4 \mathrm{~cm}$. of tissue. They were small mattress sutures including sufficient of the delicate tissue, gathering the tissue up in the grasp of the curved needie, where no blood-vessels were present, to insure against their tearing out. Four or five sutures, about one inch apart, were introduced from right to left and caught in a hemostatic forceps. The second and third row of sutures were introduced in a similar manner, the third row including the tissue of the ligament near the attachment to the liver above, and below grasping the tissue just above the blood-vessels of the lesser curvature of the stomach. The sutures were strictly confined to the normal ligamentary supports and the distance between the rows from left to right was increased with the length of the ligament, being greater toward the right. I need not point out that the important blood-vessels in relation with the right border of the ligament are to be carefully avoided, so that they are not injured or constricted in the suturing. The gauze sponges were then removed, the stomach brought into the wound and carried upward toward the diaphragm as the first, second and third row of sutures were tied. The stomach, particularly the pyloric end, was thus elevated to about normal position. If, after the sutures were secured, there remained any portion of the stomach abnormally low or there was a depression or kink along the lesser curvature, this was corrected by an additional suture. The abdomen was then closed by the layer suture method, combined with two or three silkworm gut through-and-through sutures.

The plan of operation described is that followed in the early cases in which I have operated, with the belief that it was necessary, in order to gain a broad surface of adhesion and a strong support, so as to prevent cutting out of the sutures and a recurrence of the displacement, that the ligaments be shortened by folding on themselves and that they be secured with three rows of sutures. With growing experience, however, I have verv much simplified the operation in the last eighteen cases by introducing but a single row of sutures from above downward across the gastrohepatic omentum. The strength of the suture support and attachment above has been increased by introducing each suture through the distinctly thicker and stronger one-fourth of tissue forming the attachment of the ligament to the liver. The gastrohepatic ligament at the attachment to the liver for a distance of one-fourth to one-half inch is found formed into a strong, white connective tissue band, a structure holding the suture without danger of cutting out and forming a fixed point above against which to draw the lesser curvature of the stomach.

The first suture is introduced, beginning above, in the strong tissue of the attachment of the ligament to the liver, that described, the needle including considerable tissue, and then grasps the more delicate tissue at short intervals from above downward until a position just above the gastric vessels is reached at the lesser curvature. Four to six such sutures are inserted in this manner, being perhaps an inch apart.

The suturing toward the right, opposite particularly the prolapsed pyloric end of the stomach, is carried as low and as high in the liagment as is possible, well avoiding the vessels and ducts at the right border, so as to insure the greatest elevation where it is most indicated.

When these sutures are secured the stomach, the lesser curvature, is carried in contact or almost in contact with the under surface of the liver at the attachment of the gastrohepatic ligament and is firmly fixed in this position. It is apparent to the operator at the termination of the operation that it would require considerable tearing force to displace the organ again and it is evident that a recurrence of the ptosis cannot occur.

The modification also secures a much higher position of the stomach than was possible by the original plan of suturing. I seek to have the greater curvature of the stomach above the umbilicus, at the point as demonstrated by the $x$-ray in the normal position of the stomach.

The operation is easily accomplished in twenty to thirty minutes, and in no instance has been associated with any degree of shock. There have been no deaths or complications following. The convalescence is easy, the patients being particularly free from nausea and vomiting, and the suffering is not greater than after a simple exploratory celiotomy. The bowels are opened at the end of forty-eight hours by enema. The patient is kept on small amount of liquid food for two or three days, then given semiliquid food and quickly solid food. $\mathrm{He}$ is confined to bed in the recumbent position for two weeks.

The principle of this operation is that by placing interrupted sutures from above downward through the gastrohepatic omentum, or gastrohepatic and gastrophrenic ligaments, the normal ligamentary supports of the stomach are shortened and the stomach elevated to normal position without in the least disturbing the physiologic mobility of the organ. With the elevation of the stomach any ptosis of the transverse colon is likewise corrected to a considerable-I believe sufficient -extent.

A report of the first operation performed by this method was made before the Fellows of the College of Physicians of Philadelphia by Dr. Alfred Stengel and myself on April 5, 1899. I published accounts of further experience ${ }^{\mathbf{1 0}}$ in February, 1903, and October, 1904.

Since the description of my first case Bier ${ }^{1 i}$ of Greifswald, Germany, has reported four cases in which the operation was done in a somewhat similar manner. Three or four sutures were placed through the gastrohepatic ligament from left to right so as to form two plications, one above the other, in the ligament. The first suture attached the serosa and muscularis of the pyloric end of the stomach, at the lesser curvature, to the capsule of the liver, the other included only the ligament. Also, in two of the cases the shortening of the gastrohepatic ligament was supplemented by the passing of two sutures through the serosa of the lesser curvature of the stomach and then through the capsule of the left lobe of the liver.

10. Beyєa : Univ. Penn. Med. Bull., February, 1903; Am. Med.,

11. Bier: Deutsch. Ztschr. f. Chir., 1900, lvi, 374. 
In the operation of Duret, Röysing and Gelpke the stomach was firmly fixed to the anterior abdominal wall by the passing of sutures through the serosa and muscularis of the stomach and the various layers of the abdominal wall. The fixation of the stomach to the anterior abdominal wall by adhesion, particularly to the extent practiced by Rövsing, would seem, at least in part, to destroy or hinder the physiologic mobility of the organ and result, in a greater or less number of cases, in a disturbance of the function of the organ. Likewise there is the danger of the production of the very painful affection frequently seen associated with adhesions to this organ.

The suturing of the lesser curvature to the liver and the greater curvature to the anterior abdominal wall, as practiced by Eve and LaPlace, is subject to the same criticism. Further, in a patient so operated on who atterward came under my care, pneumonia developed as a postoperative complication, and from the constant coughing a large hernia of the stomach resulted. That the greater curvature of the stomach was anchored to the abdominal wall below the incision, I believe, was an important factor in the production of this ventral hernia.

The operation of Davis, attaching the lesser omentum near the upper curvature of the stomach to the abdominal wall, would seem a better procedure, but the stomach would be distorted and abnormal adhesions formed.

The securing of the greater omentum at a point just below the transverse colon to the anterior abdominal wall one inch above the umbilicus, the operation of Coffey, elevates the stomach to a considerable extent and relieves any dragging on the stomach from below caused by the transverse colon and great omentum. Another point of possible value, according to Sailer and Clark, is that this operation corrects the associated ptosis of the transverse colon, and that it relieves the constipation from which they believe these patients suffer. The operation, however, markedly changes the anatomy of the stomach and transverse colon, and, since an artificial adhesion is formed between the great omentum and anterior abdominal wall, there would be the danger of more extensive adhesions being formed than is planned, involving the transverse colon and stomach and resulting in an incurable condition. Intes. tinal obstruction is a very possible complication. I am, however, inclined to believe that the operation might be of value and warrantable where there existed extreme hepatoptosis, the liver being so low in position that, although the lesser curvature of the stomach were brought in contact with the under surface of the liver, the stomach would still remain vertical in position and the duodenal kink persist to cause symptoms. Here it would be a more simple operation than any hepatopexy devised.

As to the question of ptosis of the transverse colon causing constipation and necessitating greater elevation of the colon than is gained by bringing the lesser curvature of the stomach in contact with the liver, I have sought information by written communication. I have replies from 23 of the patients operated on. Seven state that they did and 16 that they did not suffer from constipation before operation, and 6 do and 17 do not now suffer from constipation. Therefore, in my experience, constipation has not been a sufficiently prominent symptom to warrant the additional operation, certainly not that of resection of the colon, as is recommended by
Clark. I did elevate the colon by this method in one instance, because the patient complained of pain over the prolapsed colon aiter the stomach had been restored to position. In another case in which marked ptosis of the colon existed, the kink was corrected by simply narrowing the gastrocolic omentum over this area, thus bringing the colon almost in contact with the stomach. I have, however, noted no special advantage of the operation.

Regardless of the theoretical criticisms of the fixation operations, it is to be noted that from the published reports of the operators who have performed them, in a total of 82 cases, no such disturbances of function of the stomach or other complication is mentioned, except in an early case of Rövsing's. Rövsing, now with an experience of 75 cases, 50 of which he has had an opportunity to observe from one to eight years, asserts that none of his patients have after operation had retention of food or lave developed a disturbed function of the organ. Nyrop, ${ }^{12}$ however, discredits the good results reported by Rörsing, saying he has observed and reported cases in which such adhesions did produce pain. I have had communicated to me verbally the history of one such case in which the patient, because of the fixation operation, became a hopeless invalid. Thus, even with the small amount of clata available, it is evident that the forming of adventitious adhesions to a hollow viscus, directly or indirectly, is bad surgery and conducive to harmful results.

The operation I have devised, as I have said, simply shortens the anatomic ligamentary supports of the stomach, elevates the organ to a position where it is in contact with the liver at the point of attachment of the gastrohepatic ligament, and thus the anatomic conditions of normal mobility and physiologic function are completely preserved. The operation must, therefore, be considered anatomically, physiologically and surgically ideal.

Since the report of the first operation in April, 1898, I have operated in 25 additional cases; in all I have performed the operation 26 times. Martin, employing this method, has operated in 5 cases, Noble and William Mayo each 2, and Hodge 1. Martin, in one of his cases, also performed the Coffey operation, and in both of Mayo's cases Finney's pyloroplasty was performed. Including the four cases in which Bier operated, the operation has been performed 39 times.

All of the patients coming under my care had suffered with the characteristic and severe symptoms of gastroptosis for from three to fifteen years. In 4 a right prolapsed kidney had been operated on without relief of symptoms, and in 3 cases a floating kidney still exists, not causing symptoms after the reposition of the stomach. Regarding the presence of hepatoptosis I am unable to make any definite statements. Of the last 14 cases the skiagraph showed some ptosis in 10 , and in one the descent of the liver was extreme. In this latter case, skiagraphed several months after operation, the stomach was seen with its lesser curvature well up beneath the liver, but still in a prolapsed position because of the hepatoptosis. I believe that more or less hepatoptosis was present in all of my cases. Dr. Henry Pancoast of the $x$-ray department of the University of Pennsylvania, suggesting that the greater part of the ptosis of the liver is due to a right lateral downward rotation of this organ, in the last two cases in which we have operated I have sought to correct this by suturing

12. Nyrop: Ann. de chir. Gastro-intest., April, 1907. 
the round and falciform ligaments to the upper end of the abdominal incision, thus carrying the liver upward and toward the left. No second skiagraphs have up to the present time been made in these cases.

Twenty-five of the patients were greatly emaciated, weighing between 77 and 120 pounds. In 16 the upper curvature of the stomach was outlined by means of the skiagraph or by inflation and auscultatory percussion one and $a$ half to two and a half inches below the umbilicus, in 9 three inches below the umbilicus (the stomach reaching well into the pelvis), and in 1 one inch below the umbilicus. The latter was a small tubular stomach, vertical in position, the $x$-ray showing a marked kinking in the duodenum. In three an associated dilatation of the stomach was diagnosed, but, as is common, the dilatation was not appreciable at operation. In two there was a pronounced dilatation of the stomach demonstrable at operation. In none of the cases was there present a diastasis of the recti muscles or great relaxation of the abdominal walls, although seven of the women had borne children. All of the patients operated on suffered from retention and fermentation of food, were chronic invalids and most of them incapacitated for any occupation or social duty. Operation was determined on in every instance only after a long course of medical and mechanical treatment had been carried out by a competent internist and the treatment had failed to give permanent relief. Several had been transferred to the care of an internist for the purpose of giving the medical treatment a thorough trial. In every instance the patient had been treated faithfully with medicine, hygiene, diet, bandaging, binder or a corset, and the majority had had a six weeks' rest cure. They were all willing and anxious because of their suffering to risk the danger of operation for the sake of a possible cure.

In one instance in which the stomach was prolapsed so that the upper curvature was two inches below the umbilicus and the organ dilated to perhaps twice its normal size the woman was emaciated until she weighed but $7 \%$ pounds. Further, she apparently suffered from autointoxication as a result of the gastric and intestinal fermentation process, to such a degree as to produce every two or three weeks a rise of temperature reaching 102 to $103 \mathrm{~F}$. She was in extreme condition, the operation dangerous, but demanded by the family physician. This patient has gained 30 pounds in weight, has become pregnant and borne a child since, all symptoms have disappeared and she enjoys perfect health. Six of the patients were males, one a physician who had given up practice and had devoted several years of his life to seeking relief in various parts of the country.

The 26 patients on whom I have operated have now been observed from four weeks to eleven years. In orcler to gain an accurate report as to the result of the operation I have recently communicated with these patients by circular letter. A reply was received from all.

In every instance, except Patients 11 and 18 and one patient recently operated on, the improvement in health had been most remarkable and the relief of symptoms complete. The dyspeptic and neurasthenic symptoms are entirely relieved, there is no retention or fermentation of food, and food of any character is taken without restriction. All have gained in weight from 8 to 40 pounds, the average gain in weight being 20 pounds. The gain in weight and state of improved health has been maintained regardless of the time that has elapsed since operation.

Patient 11 was operated on in June, 190\%. She immediately gained ten pounds in weight and was relieved of her symptoms. She then relapsed into the old condition, suffering as much as before operation and now practices lavage daily and before going to sleep at night it is necessary that her physician replace a prolapsed right kidney. Preceding operation she was under the care of Dr. John K. Mitchell at the Orthopedic Hospital, who carried out a thorough rest-cure and medical treatment without the least benefit. 1 had previously refused to operate on this woman, but, it being again suggested by Dr. Mitchell because of the failure of the rest cure, the operation was carried out, although I felt at the time that there was little chance of its giving relief. This also is the case referred to in which there was an extreme hepatoptosis.

Patient 18, operated on in March, 1908, a young man of no occupation, is free from symptoms referable to the stomach and transverse colon, both being elevated, and

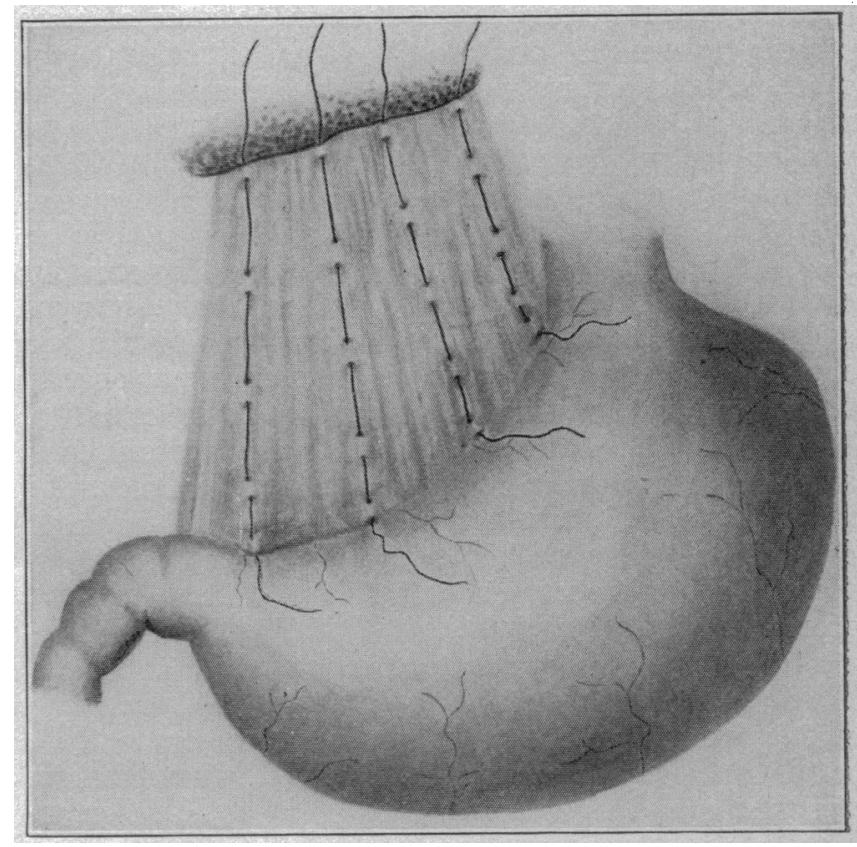

Suture of the gastrohepatic omentum to secure elevation of the stomach in gastroptosis.

has gained 10 pounds in weight, but still complains of pain in the lower abdomen. It is his belief that this pain is due to a separation or tearing away of the mesentery of the small intestine, following a severe strain.

In Case 4 the patient was markedly benefited for a time, and then after nursing two sisters through a course of typhoid fever became profoundly neurasthenic; after a rest-cure she is again enjoying good health.

These three cases are simply instances in which there exists, associated with the gastroptosis, essential neurasthenia, as occurs with other conditions and disease. 'The patients are little, if at ali, benefited by medical or surgical treatment and are sometimes made much worse by the latter.

The patients operated on by Martin gained from 20 to 30 pounds in weight and are relieved of their previous symptoms. Those operated on by Noble, I am told, are relieved of their symptoms. The patient whose case was described by Hodge I saw a short time ago and 
she has gained in weight and has good health. The four patients operated on by Bier have all gained in weight, from 11 to 25 pounds, and, he states, enjoy good health.

The immediate and lasting complete relief of symptoms, improvement in nutrition and gain in weight in all but two of the 39 patients operated on by simply shortening the gastrohepatic omentum would prove that the gastric symptoms were induced by the abnormal position of the stomach in gastroptosis, and that the neurasthenia is, as a rule, not an essential but an induced condition. The completeness of relief and remarkable benefit following invalidism which had existed for years in 95 per cent. of the cases, the simplicity of the operation (which restores the stomach to normal position without removal of tissue or the formation of abnormal adhesions) and the fact that it is practically free from danger (we would estimate the danger as not being more than one-fourtl of 1 per cent.) must strongly recommend this surgical treatment in every case of gastroptosis in which the suffering is great.

It has been my experience that in no class of cases coming to me for treatment do the pationts receive more benefit or are more grateful than those here referred to. The mechanical and medical treatment at best only secures partial relief of symptoms. (The $x$ ray las now positively determined, as stated by Pancoast, Sailer and Worden, ${ }^{13}$ that no form of binder, bandage or corset elevates the stomach to the smallest extent.) It entails constant treatment, diet, hygiene and the wearing of a cumbersome bandage or irritating adhesive straps, while the operation promises at once to restore completely and permanently the patient's health.

It is true that the patients thus far operated on represent but one class, those with gastroptosis occurring independent of relaxation of the abdominal walls or diastasis of the recti muscles. Experience alone can teach what influence the operation can have where a relaxation of the abdominal wall is active in the production of the displacement of the stomach. I believe, however, that the new attachment would be sufficiently strong to maintain the organ in normal position and the result would be quite as satisfactory. I do not believe that the opcration devised by Webster would be necessary.

In cases in which there exists an associated and even marked dilatation of the stomach, as in two of our cases, the dilatation is dependent on the gastroptosis with kinking of the duodenum and motor insufficiency of the gastric walls, and therefore with the relief of the kink or obstruction through the elevation of the stomach the stomach drains itself, the motor activity is restored and the dilatation must soon disappear. Such is amply proved in my case in which dilatation was extreme, caused autointoxication and the patient after operation gainer 30 pounds in weight. The stomach has returned to normal size.

I see no indication for the combination of pyloroplasty with elevation of the stomach or the performance of pyloroplasty alone in gastroptosis, unless there be present a true organic stricture of the pylorus. The operation of gastrojejunostomy as practiced by Walker, Hammer and Dcaver I would consider unnecessarily mutilating, dangerous and in no sense indicated in this disease.

13. Worden: Tr. Coll. Phys., Philadelphia, 1906, Series 3, xxviii, 151-196.
1 would again call attention to my experience that in performing the operation the stomach cannot be carried too high upward beneath the liver to relieve the duodenal hink and compensate for the coexisting ptosis of the liver.

i he operation here described has been subjected to a certain amount of criticism by three writers. It is stated by Rövsing and Eve that the gastrohepatic omentum is such a frail, delicate tissue that sutures cannot be introduced into it, that even though sutures were thus introduced they would cut out and the displacement of the stomach recur, and that the operation therefore is an impracticable one. It likewise appears that Bier, Noble and Lallace in performing the operation were not satisfied to rely on the simple suture of the gastrohepatic omentum alone, but supplemented. the opseration by passing sutures between the lesser curvature of the stomach and capsule of the liver, or greater curvature and abdominal wall. The gastrohepatic omentum is thin and delicate; in two of our cases particularly it was very thin, but, as far as my experience gocs, in all 26 cases there las been no difficulty in finding sufficiently strong tissue not only to hold the sutures but to make a much niore than efficient elevation and attachment of the organ, particularly with the modification here described. As stated, in every instance it would have required much tearing force at the termination of the operation to separate the stomach. A still better proof is the fact that there have been no recuriences.

Rörsing and Deaver have offered the criticism that, because of the presence of numerous blood-vessels, particularly veins, in the gastrohepatic omentum, these vessels would easily be injured or constricted and the operation made a dangerous one. The field of operation is plainly before the operator, the gastrohepatic omentum transparent, and therefore these vessels are readily seen and avoided, and only a very careless operator would endanger the life of his patient through such complication. Also, no important vessel is ever constricted if the operation is carried out with ordinary care.

Finally, I wish to point out that there exists in gastroptosis a physical displacement of a most important organ; the whole cause of the patient's illness results from this mechanical displacement; or in a general visceral ptosis it is the stomach which causes the greater part of the symptoms. The gastromotor insufficiency, the dilatation and their symptoms are the direct result of the malposition of the stomach. The medicinal and mechanical treatments give relief in a large percentage of cases, the severe symptoms disappear and, I might say, the patients are comfortable. This relief is gained by constant careful feeding, regulated rest after taking food or the full rest treatment and the wearing of a bandage or application of adhesive straps. 'The binder gives relief by increasing the intra-abdominal pressure and not by elevating the stomach. With the removal of the binder, the taking of food of normal amounts and character and the assuming of any occupation, the symptoms gradually return, for the cause of the suffering is in no sense relieved. At least this has been my experience in every instance in which the patient was first referred to an internist for treatment. Therefore, if the suffering of the patient is great, I can only come to the conclusion that the surgical treatment is the only logical one.

1734 Spruce Street. 